\title{
LUMBAR SEGMENT DURING THE WALK
}

\author{
Z. Sant *, L. Mifsud ${ }^{* *}$, C. Muscat ${ }^{* * *}$
}

\begin{abstract}
Designs of medical devices are tested for their mechanical behaviour, and ability to transfer the load that is normally bore by the healthy tissue. The virtual testing in silico is widely accepted technique and will be used in our work to simulate a walk. The already existing Finite Element (FE) model of the lumbar spine was reused while the virtual behaviour was controlled by a set of constrains and a load data, normally transferred through the load-bearing tissue. The magnitude and character of the used load depended on the physical activity, external loads, physical condition of the subject and its specific factors such as gender, health condition, etc. Most of the published simulations used estimated simplified loads that barely simulate the real behaviour. This paper will discuss performance of the lumbar segment during the walk. The musculo-skeletal system is a statically indeterminate system thus the forces were solved via computational modelling using the trajectories recorded by VICON system, and consequently exported into an open software for computation. All forces acting on the segment were exported to into Finite Element (FE) database and the obtained results presented the importance of correct material model as well as the corresponding load model.
\end{abstract}

Keywords: lumbar spine, muscular activity, finite element

\section{Introduction}

Human mobility is commonly taken as a matter of course, until the first symptom of musculoskeletal disorder, demonstrated as a low back pain (LBP) occurs. The low back dysfunction is associated usually either with chemical irritation or tissue damage due to mechanical stretching of spinal connective tissues. There are a number of studies addressing LBP treatment; unfortunately they do not present any conclusive results as the causality is usually very complex with large number of interacting factors. In this work we will concentrate on the biomechanical feedback of the lumbar spine segment to loads generated during the walk. The fact that almost every adult experienced LBP at least once in his life, shows the necessity to learn more about the loads, and response of the tissues at this location. It is impossible to measure the response, and the loads generated during the walk directly in vivo. Therefore we used computational approach, and existing virtual lumbar segment to simulate the spine behaviour during the walk, as the human common activity. It was reported that in one year the lumbar spine is exposed to approximately 3 millions of cycles, of a person with an average physical activity ( 5 times per week of 30 minutes at moderate-intensity activity), while in case of the ones who are active in sport, it can reach up to 8 million cycles (WHO, 2018). This implies the importance of the healthy lumbar spine, and any new views and facts about its behaviour can through more light on the crucial factors that might play its role in LBP. It is assumed that biochemistry, and mechanical forces are associated with LBP, but the degeneration process is still not well described, and even the factor initiating this process is not known. In this work we concentrated on the mechanical factors such as density of bone, the architecture of the cancellous bone, the thickness and condition of end-plate tissue, and the effect of the mechanical load

\footnotetext{
* Dr. Ing. Zdenka Sant: Mechanical Engineering Dept., University of Malta, Tal Qroqq; MSD2080, Msida; MT, zdenka.sant@um.edu.mt

** Louise Mifsud, B.Eng.(Hons.): Department of Mechanical Engineering, Imperial College London, Kensington, London SW\&2AZ, UK, loumif@gmail.com

*** Carl Muscat, B.Eng.(Hons.): Department of Biomedical Engineering, University of Strathclyde, 16 Richmond Street, Glasgow G1 1XQ. Scotland, UK, carl.muscat.10@um.edu.mt
} 
acting on the lumbar segment tissues, using Finite Element (FE) model of lumbar spine segment L3-L4. The FE model is based on the geometry obtained via segmentation of CT scans of 15 year old male subject. The FE material model, as shown in Fig. 1, was initially assigned to have isotropic properties, then was remodelled based on the micromechanical characterization of CT scan (Blanchard, 2013) into the orthotropic material properties corresponding to grey values of the images.

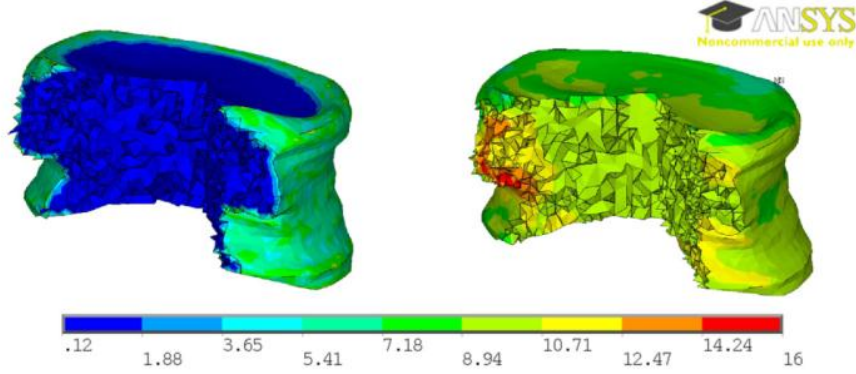

Fig. 1: Contour plot of Young's modulus (GPa) throughout a cutaway of vertebra with isotropic and orthotropic material model respectively

The mechanical load factor and its consequence on the lumbar segment are being discussed in this paper. The strength of the spinal column is reinforced by posterior and anterior longitudinal ligaments that are directly attached to the vertebrae, ligamentum flavum, and interspinous ligaments. The extensor, flexor, lateral flexor, and rotator are muscles actively supporting the movement, and contribute to the spine stability. These muscles are overlapping each other internally, which prohibits the direct measurement of the force magnitude.

\section{Methods}

The size of the muscle, mainly the cross-section area, effects the muscular force potential while muscle attachments determine the muscle's line of action that together with a moment arm ascertains the production of moment, and stabilization. The direct evaluation of muscular forces is possible by means of electromyography technique that is not efficient for simultaneous analysis of groups of muscles. In these cases computational modelling techniques are more feasible to use.

\subsection{Computational modeling of muscle activity}

The fundamental problem of computational approach is related to the higher number of muscles than it's necessary for creation of specific movement, thus the solution leads to infinitely many patterns of a muscle recruitment that satisfy the dynamical condition (Damsgaard et al.,2006). The statics and dynamics equations (1) provide solution of reaction forces and moments at the joints.

$$
\mathbf{C} \mathbf{f}=\mathbf{d}
$$

Where $\mathbf{C}$ represents the matrix of coefficients within the static equations that are dependent on the position, $\mathbf{f}$ is a matrix of muscular and reaction forces, and $\mathbf{d}$ is a matrix of external and inertia forces. To obtain the results that correspond to the muscle recruitment, an optimization has to be introduced to the system of dynamic equations, with the objective function $G\left(f^{(M)}\right)$ that minimizes the muscular forces, subject to a system of static equations (1) with the prescribed condition

$$
0 \leq f_{i}
$$

Defining $f_{i}^{(M)}$ as the force of $\mathrm{n}$-th muscle in the system while $N_{i}$ represents the normalizing factor defining typical muscle strength. The adopted objective function called the Min/Max criterion (3) leads to a distribution of muscular forces that keeps maximum relative load to minimum.

The active muscles control the motion of each vertebra thus it is of utmost importance to simulate the muscle recruitment that mimics the reality, especially on the lumbar vertebrae. Components of the erector spinae connect to the mamillary, accessory, and transverse processes of the lumbar vertebrae. Their line of action is not parallel to the compressive axis of the spine, but has rather a posterior-caudal obliquity that causes a posterior shear force together with an extensor moment on the superior vertebrae. The 
multifidus muscle spans over few joints thus it's involvement is local, to correct or support extensor torque at specific joints that may be foci of stress (Herzog, 2000).

\subsection{Simulation of spine behaviour}

Prior to spine behaviour simulation, a young male with corresponding anthropometric characteristic of the $\mathrm{CT}$ scan subject was selected, and the motion data was recorded in c3d format to be imported into the open software AnyBody (AB). Prior to data processing, the AB existent generic musculoskeletal model with markers was scaled to correspond to our subject's anthropometric data. In the next step the L3-L4 vertebrae of the generic model $\mathrm{AB}$ were morphed to obtain the size and shape that corresponds to L3-L4 vertebrae of the subject's CT scan as shown in Fig.2, and the morphed vertebrae were imported back into the AB generic model. The response of the lumbar segment was evaluated by means of FE Analysis (FEA), the tool for computational simulation of a response to different loads and material models.
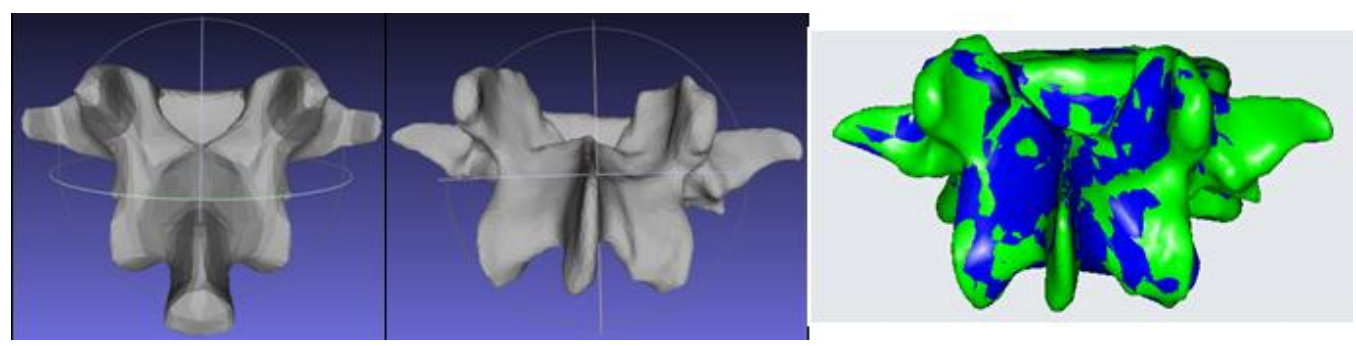

Fig. 2: GenericL4.stl, TargetL4.stl respectively, and morphed L4 Generic Blue \& Target Green

Creation of the load model included identification of all points of tendons and ligaments attachment that were marked on the vertebrae surface and used to apply the forces obtained from $\mathrm{AB}$. Then the $\mathrm{AB}$ model with morphed vertebrae and the selected optimization function $\mathrm{max} / \mathrm{min}$ processed all imported data in c3d format by means of inverse kinematic and dynamics. All results of required muscular forces were exported from $\mathrm{AB}$ in XML format to be read by a subroutine AnyFE that transferred data into the FE package ANSYS. The uploaded existing geometry model of the lumbar segment with following properties of cortical and cancellous bone of $12 \mathrm{GPa}$ and $150 \mathrm{MPa}$ respectively were meshed with SHELL181 elements of thickness $1 \mathrm{~mm}$ and SOLID185 respectively, endplates with $\mathrm{E}=500 \mathrm{MPa}$ were meshed with SHELL181. Orthotropic material properties applied to advanced model ortho, simulated subject specific cancellous bone of varied properties between 0.12 - $16 \mathrm{GPa}$. Both segment models iso and ortho simulated ligaments and tendons transferring the forces to vertebrae by BEAM4 with Young's modulus of $15 \mathrm{MPa}$ (Ibarz, 2013). Prior to running a code of instruction AnyFE, to translate the XML data to ANSYS Parametric Design Language (APDL), the coordinate system was aligned to correspond the one used in AB. Then the APDL code created a local coordinate system for each muscle/ligament insertion, and computed force components were applied to the corresponding points followed by the connection between the force and the selected set of nodes on the bone surface. The boundary condition constraining the inferior endplate of the inferior vertebra L4 was set. The connection between adjacent vertebrae and IVD was simulated by sets of constrain equations for inferior and superior connection.

\section{Results}

Two groups of results were obtained in this study. The first force results, computed via optimized inverse dynamics, were plotted along the whole gait cycle and the trend of muscle recruitment was validated against the trend of 'typical activity of major muscle groups' during gait (Winter, 1989). A total of nineteen forces ranging from -3.4 to $31.2 \mathrm{~N}$ acting on each vertebra were applied as a single force model component of the load in the FEA for both material models, iso \& ortho. Muscle model was able to exert only tensile forces. No tendons or passive element properties were modeled and no muscle dynamics was considered such as force-length, and force-velocity relationships. The variation of stress/strain magnitude depends on the position and phase of the gait cycle as shown in Fig. 3. The attention was paid to identified locations of interest - pedicle area and endplates. The stress intensity in L3 vertebra, due to AB load, varied along the pedicle axis in posterior-anterior direction for iso and ortho model from $0-1.2$ $\mathrm{MPa}$ and $0-0.2 \mathrm{MPa}$ respectively. The comparison between the ortho and iso models loaded by AB forces show the highest discrepancy of the stress intensity at the centre of endplate of $0.024 \mathrm{MPa}$ and $0.636 \mathrm{MPa}$ respectively. The total displacement at the centre of superior endplate reached $0.133 \mathrm{~mm}$ and $0.117 \mathrm{~mm}$ for ortho and iso model respectively. 
Stress on cancellous bone v.s. Gait cycle percentage for posterior node

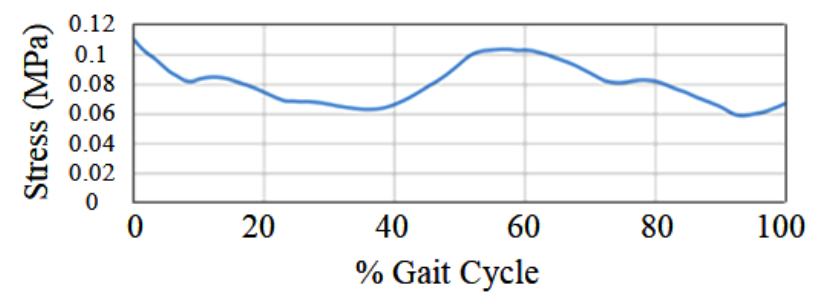

Stress on cancellous bone v.s. Gait cycle percentage for anterior node

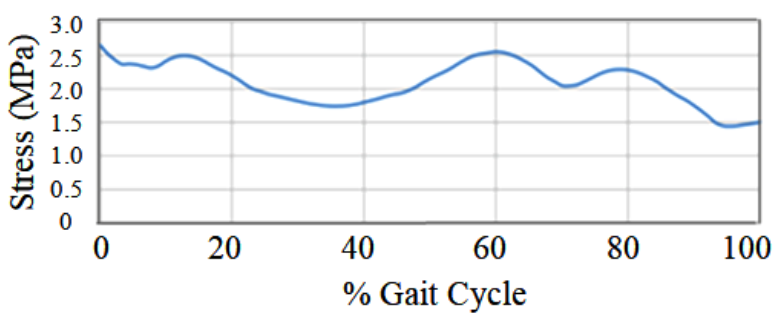

Fig. 3: Stress variation at the posterior/anterior point of L3 vertebra with isotropic material model

\section{Conclusions}

The presented results are still not final thus we can draw only a partial conclusion. The results from the load step simulating the heel strike were first compared to the previous computation simulated by the normal and shear force accompanied by flexing moment, all applied at the centre of IVD, as shown in Fig. 4. The iso model suggests that uniform model properties cannot really predict the response close to the real situation since the redistribution of the load within the cancellous bone depends of the bone architecture that is never uniform due to mechanical load transfer.

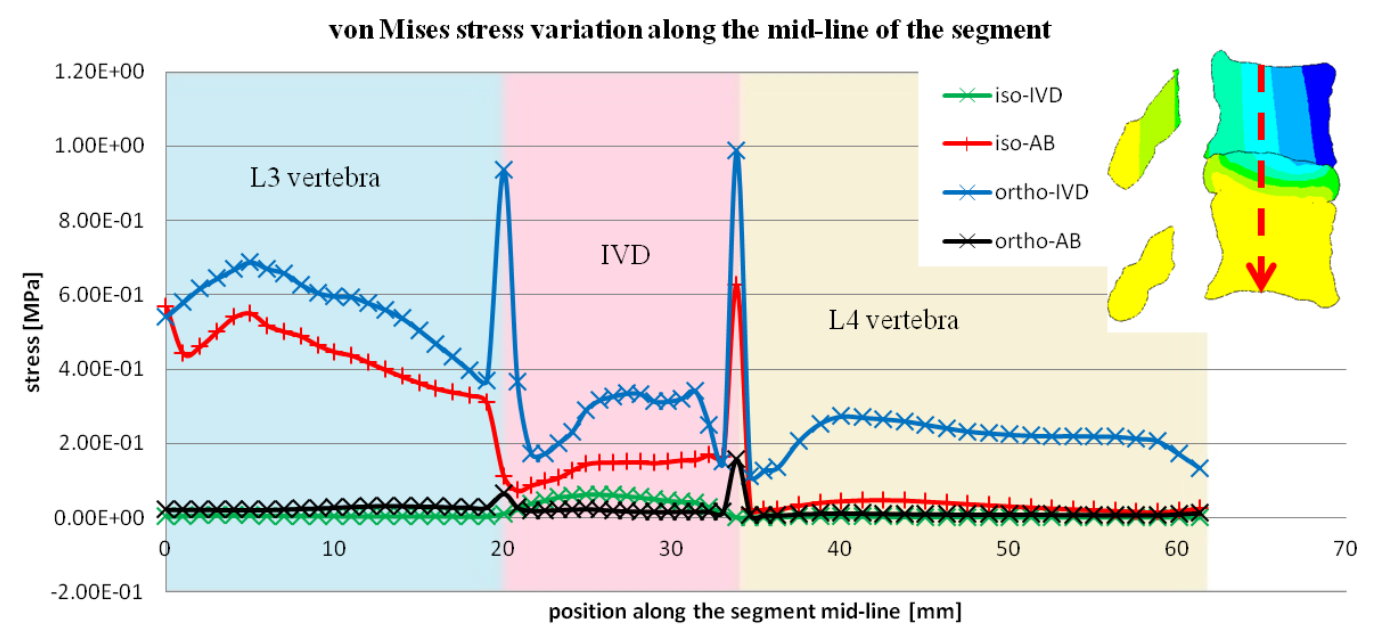

Fig. 4: Comparison of stress intensity along the mid-line of the segment

\section{Acknowledgement}

This work was supported by the Research Ethics Committee with their permission, and Radiology Department, Mater Dei hospital, Malta providing access to CT scans. Further gratitude goes to all collaborators from the COST Action MP1005: NAMABIO for support and contribution to this project, and kind support of BioPark Lab, and OTH Regensburg, Germany,

\section{References}

Blanchard, R., Dejaco, A., Bongaers, E. \& Hellmich C. (2013) Intravoxel bone micromechanocs for microCT-based Finite Element simulations. Journal of Biomechanics, 46(15), pp. 2710-2721.

Damsgaard, M., Rasmussen, J., Christensen, S. T., Surma, E., de Zee, M. (2006) Analysis of musculoskeletal systems in the AnyBody Modeling System. Simulation Modelling Practice and Theory, 14, pp. 1100-1111.

Herzog, W. (2000) Clinical Biomechanics of Spinal Manipulation, Churchill Livingstone

Ibarz, E. et al (2013) Instability of the lumbar spine due to disc degeneration. A finite element simulation. Advances in Bioscience and Biotechnology, 2013, 4, 548-556

WHO (2018) Physical Activity and Adults, http://www.who.int/dietphysicalactivity/factsheet_adults/en/

Winter, D. A. (1989) Biomechanics of Normal and Pathological Gait: Implications for Understanding Human Locomotor Control. Journal of Motor Behavior, Vol.21(4), p.337-355 